\section{Blick in die Zukunft: Der Traum von der perfekten Wundheilung}

Bisher hat die Narbenprophylaxe und -therapie das Ziel einer Wundheilung mit einer möglichst unauffälligen, normalen Narbe. Doch Experten erlauben sich inzwischen auch schon mal den Traum von dem Heilungsergebnis einer normalen Haut.

$\mathrm{E}$ inen solchen Optimismus nährten Ergebnisse mit der Substanz Avotermin im letzten Jahr. Dabei handelt es sich um einen rekombinanten Wachstumsfaktor, dem Tumorwachstumsfaktor-Beta3 (TGF- 33 ) [Ferguson MW et al. Lancet 2009; 373: 1264-74] - dem Schlüsselzytokin für die narbenlose Abheilung in der Embryogenese, wie Prof. Dr. Sigrid Karrer aus Regensburg betonte. Die Haut von Erwachsenen weist dieses Zytokin nur noch in geringen Mengen auf. In 3 doppelblinden, placebokontrollierten Phase-I/II-Studien injizierten die Untersucher deshalb prophylaktisch in frische $1 \mathrm{~cm}$ lange Wundränder randomsiert Avotermin oder Placebo. Ansonsten erfolgte in beiden Studienarmen jeweils dieselbe Standard-Wundversorgung.

Bei Verwendung des rekombinanten TGF- $\beta 3$ heilten die Wunden signifikant schneller und die Narbenheilung verbesserte sich anhaltend - teilweise sah man kaum mehr eine Narbe, berichtete Karrer, und betonte dabei die gute Verträglichkeit der Behandlung. Das Konzept wird deshalb weiter verfolgt: Derzeit läuft in 9 europäischen Ländern und den USA eine Phase-III-Studie an 350 Patienten nach einer Narbenrevisions-Operation, eine
Pilotstudie untersucht derzeit außerdem die Wirksamkeit der Substanz zur Rezidivprophylaxe nach der Operation von Ohrkeloiden. Karrer meint optimistisch: „Sollte sich tatsächlich bestätigen, das man mit TGF- $\beta 3$ die Rezidive von Keloiden verhindern kann und dann auch noch die Narben narbenlos abheilen, können wir unseren Patienten vielleicht in Zukunft sagen, dass sie nicht nur eine normale Wundheilung erwarten können, sondern sogar eine normale Haut."

Doch bei allem Optimismus: Im Augenblick empfiehlt sie dringend, Patienten immer klar zu sagen: Ziel der Narbenprophylaxe und Narbentherapie ist eine normale Narbe, die kosmetisch akzeptabel ist, nicht juckt und spannt, keine Schmerzen bereitet und nicht rezidiviert, nicht aber das komplette Verschwinden aller Zeichen der früheren Verletzung!

Kurs „Probleme mit Narben - aktuelle Therapiemöglichkeiten". 27.7.2010.

\title{
Aussichten für die Ästhetische Dermatologie
}

\section{Der Markt für Anwendungen in der Ästhetischen Dermatologie ist sehr unübersichtlich - evidenzbasierte Verfahren sollten bevorzugt werden.}

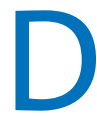
ie ästhetische Medizin sollte nach Ansicht von Prof. Dr. Berthold Rzany, Dermatologe an der Charité in Berlin, stärker in die Aus-, Fortund Weiterbildung integriert werden. In den vergangenen Jahren habe sich in diesem „anfälligen Bereich“, so Rzany, sehr viel getan, ohne dass immer eine wissenschaftliche Expertise vorhanden sei. Die ästhetische Medizin sollte sich deshalb von Behandlungen, die nicht evidenzbasiert sind, absetzen.

\section{Botulinumtoxin bald topisch?}

Bei den Anwendungen von Botulinumtoxin in der ästhetischen Dermatologie gebe es mittlerweile mehr „Evidenz als Eminenz“, berichtete Rzany. Fragen zur optimalen Dosierung, zum Wirkungseintritt, wann das Maximum der Wirkung erreicht wird und wie lange die Wirkung anhält, können inzwischen auf der Grundlage von Studien beantwortet werden. Eine Patientin, die mit der Frage kommt, wann muss ich mich behandeln lassen, um zur Hochzeit „einigermaßen entspannt“ auszusehen, könne heute wesentlich verlässlicher als noch vor wenigen Jahren beraten werden, sagte Rzany.

Nach Rzanys Darstellung könnten eventuell schon in naher Zukunft auch topische Botulinumtoxin-Präparate eine Rolle spielen. Es gebe Firmen, die so etwas entwickeln, berichtete er: In einer kleinen Studie sei Botulinumtoxin in eine Creme gemischt und den Patienten zum Auftragen gegeben worden. Die Unterschiede seien hoch signifikant gewesen. So hätten sich beispielsweise Ringe unter den Augen oder eine Hyperpigmentierung stark gebessert. Laut Rzany könnten topische Botulinumtoxin-Präparate eine Alternative insbesondere zu den Behandlungen sein, bei denen die Infiltration sehr schmerzhaft ist.
Bei Fillern gilt:

\section{Eminenz vor Evidenz}

Anders verhält es sich nach Aussage von Rzany bei den Fillern, bei denen immer noch die „Eminenz die Evidenz“ überwiege. Aber auch hier habe sich in den vergangenen fünf Jahren viel verändert. Mittlerweile gebe es einige klinische Studien, etwa zu den Nasolabialfalten. Die dadurch gewonnenen Informationen können genutzt werden, um Patientinnen aufzuklären, wie teuer eine solche Behandlung ist und wie oft sie wiederholt werden muss.

Nach wie vor sei der Markt aber sehr unübersichtlich, räumte Rzany ein. Um erwünschte Wirkungen und vor allem seltene Reaktionen zu erfassen, sei schon vor einiger Zeit auf der Grundlage der Injectable Filler Safety-Study (IFS-Study) in Berlin unter www.derma-filler.de ein einzigartiges Register eingerichtet worden. Mit einem solchen Register sei es möglich, „verheerende“ Präparate zu verhindern, erklärte Rzany. Ein vergleichbares Register gebe es auch unter www. fillerwelt.de

sto

Plenarvortrag „Neueste Erkenntnisse: Ästhetische Dermatologie“. 27.7.2010. 